\title{
Optical activation of Be implanted into GaN
}

\author{
C. Ronning, ${ }^{\text {a) }}$ E. P. Carlson, D. B. Thomson, and R. F. Davis \\ Department of Materials Science and Engineering, North Carolina State University, Raleigh, \\ North Carolina 27695
}

(Received 30 March 1998; accepted for publication 22 July 1998)

\begin{abstract}
Single crystalline (0001) gallium nitride layers were implanted with beryllium. Photoluminescence (PL) measurements were subsequently performed as a function of implantation dose and annealing temperature. One new line in the PL spectra at $3.35 \mathrm{eV}$ provided strong evidence for the presence of optically active $\mathrm{Be}$ acceptors and has been assigned to band-acceptor $(e A)$ recombinations. The determined ionization energy of $150 \pm 10 \mathrm{meV}$ confirmed that isolated Be has the most shallow acceptor level in GaN. Co-implantation of nitrogen did not enhance the activation of the $\mathrm{Be}$ acceptors. () 1998 American Institute of Physics. [S0003-6951(98)03138-6]
\end{abstract}

Achievement and control of substantial activation of $p$ type dopants in gallium nitride $(\mathrm{GaN})$ remains a critical issue vis à vis improved performance of devices fabricated in this material. The most commonly used $p$-type dopant is magnesium $(\mathrm{Mg})$ which substitutes on $\mathrm{Ga}$ sites and has an ionization energy of $\sim 0.25 \mathrm{eV}$. One-to-two orders of magnitude higher atomic concentration of $\mathrm{Mg}$ must be incorporated into $\mathrm{GaN}$ to achieve the desired hole concentration at room temperature. ${ }^{1}$ This incorporation reduces the hole mobility due to the enhanced carrier-impurity scattering processes. ${ }^{2}$ Beryllium $(\mathrm{Be})$ is a more promising candidate for $p$-type doping given its calculated lower ionization energy of $\sim 0.06$ $\mathrm{eV}^{3}$

At this writing, Be-containing GaN films have only been grown by molecular beam epitaxy $(\mathrm{MBE})^{4-6}$ because of the unavailability of suitable Be precursors for metalorganic vapor phase epitaxy (MOVPE). High concentrations of Be have been achieved; however, the measured electrical ${ }^{4}$ and optical $^{5,6}$ properties were uncertain and contradictory. An ionization energy of $0.25 \mathrm{eV}$ was reported based on photoluminescence $(\mathrm{PL})$ spectra which were identical to those of $\mathrm{Mg}$ doped GaN. ${ }^{5}$

Doping by ion implantation offers advantages in comparison to doping during film growth. (i) The concentration as well as the lateral and depth distributions of the dopants are precisely controllable, and (ii) almost all elements can be implanted with sufficiently high purity. However, this process is compromised by the radiation damage which has to be removed via annealing. Approximately three dozen GaN ion implantation studies have been reported at present. The thermal stability, ${ }^{7,8}$ electrical activation ${ }^{9}$ of the implanted species, and the radiation damage ${ }^{10}$ were investigated as a function of annealing temperature and condition. The implanted $\mathrm{Be}$ did not diffuse during annealing procedures to $800{ }^{\circ} \mathrm{C}^{7,8}$ Photoluminescence spectra of Be implanted GaN were reported by Pankove and Hutchby; ${ }^{11}$ however, high doses were used, and only the common yellow luminescence band at $\approx 2.2 \mathrm{eV}$ was observed.

In this letter, we report near band edge PL measurements of low dose Be implanted GaN samples grown by MOVPE.

${ }^{a)}$ Electronic mail: carsten.ronning@uni-konstanz.de
Evidence for optically active $\mathrm{Be}$ acceptors was observed from a new line in these spectra due to band-acceptor $(e A)$ recombinations. These results initially and experimentally confirm the low ionization energy of Be acceptors in $\mathrm{GaN}$.

One-to-two $\mu \mathrm{m}$ thick epitaxial, monocrystalline, and undoped GaN films were grown on on-axis $n$-type, Si-face $\alpha(6 \mathrm{H})-\mathrm{SiC}(0001)$ substrates at $1000^{\circ} \mathrm{C}$ and 45 Torr using a vertical, cold-wall, rf inductively heated MOVPE deposition system. $^{2} \quad$ A $0.1 \mu \mathrm{m}$ high-temperature monocrystalline $\left(1100^{\circ} \mathrm{C}\right) \mathrm{AlN}$ buffer layer was deposited prior to the $\mathrm{GaN}$ growth. Deposition was performed using triethyaluminum (TEA) and triethylgallium (TEG) in combination with 1.5 slm of ammonia $\left(\mathrm{NH}_{3}\right)$ and $3 \mathrm{slm}$ of $\mathrm{H}_{2}$ diluent.

Beryllium was twice implanted at 100 and $200 \mathrm{keV}$ to create a broad depth distribution of this element. The dose ratio between the two implantation energies was $2 / 3$ to adjust the maximum impurity concentration of both implantations. TRIM simulations gave a mean ion range of $276 \mathrm{~nm}$ [full width half maximum $(\mathrm{FWHM})=175 \mathrm{~nm}]$ and $472 \mathrm{~nm}$ $(\mathrm{FWHM}=1060 \mathrm{~nm})$, respectively, for the two energies. ${ }^{12}$ The total implantation dose ranged between $10^{13}$ and 2.5 $\times 10^{14} \mathrm{~cm}^{-2}$. A second set of GaN samples was coimplanted with an equal dose of nitrogen to determine the efficacy of co-implantation for the activation of Be acceptors due to their occupation of the additional metal sites created by the excess nitrogen. The implantation energy of the nitrogen was $350 \mathrm{keV}$ for a projected optimum overlap of the Be profiles.

All implanted samples were sequentially annealed in vacuum at 300,600 , and $900^{\circ} \mathrm{C}$ for $10 \mathrm{~min}$ to remove the implantation damage. Photoluminescence measurements were performed after each annealing step at $14 \mathrm{~K}$ by exciting the $\mathrm{GaN}$ samples with a $\mathrm{He}-\mathrm{Cd}$ laser $(3.81 \mathrm{eV})$.

The low-temperature PL spectra of a Be doped GaN sample with a dose of $5 \times 10^{13} \mathrm{~cm}^{-2}$, measured after annealing to $900{ }^{\circ} \mathrm{C}$, is displayed in Fig. 1(a). The intensity of the band edge luminescence is several orders of magnitude lower in comparison to as-grown, unimplanted GaN samples [Fig. 1(b)]. The weak luminescence line at $3.467 \mathrm{eV}$ (commonly labeled as $I_{2}$ ) in Fig. 1(a) originates from recombinations of free excitons and/or excitons bound to shallow donors. The longitudinal optical (LO) phonon replicas for this line could 


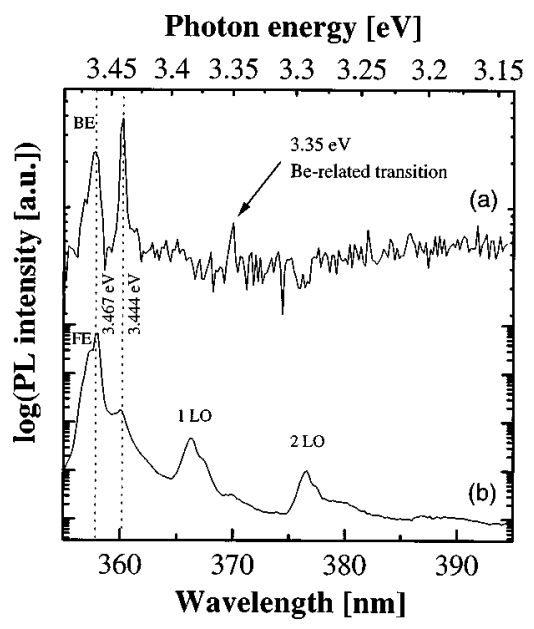

FIG. 1. Photoluminescence spectra of (a) GaN implanted with Be measured at $14 \mathrm{~K}$ and annealing to $900^{\circ} \mathrm{C}$ for $10 \mathrm{~min}$, and (b) as-grown, unimplanted $\mathrm{GaN}$. The implantation energies were 100 and $200 \mathrm{keV}$ and the total implantation dose was $5 \times 10^{13} \mathrm{~cm}^{-2}$.

not be observed in the implanted samples due to its low intensity; however, their positions at 3.384 and $3.292 \mathrm{eV}$ were determined from as-grown, unimplanted $\mathrm{GaN}$ grown on the same $\mathrm{SiC}$ wafer [Fig. 1(b)]. This results in a phonon energy of about $85 \pm 5 \mathrm{meV}$ for our samples, which is in agreement with values in the literature. ${ }^{13}$ The second luminescence peak in Fig. 1(a) at $3.444 \mathrm{eV}$ is most likely related to defects created during the implantation procedure, as this line also was observed with varying intensities after implantation of $\mathrm{Li}, \mathrm{Si}, \mathrm{Ge}$, In, and Er. ${ }^{14}$ We believe that this line is produced by nitrogen vacancies due to donor-band $(e D)$ transitions, because it appears also in unimplanted $\mathrm{GaN}$ samples depending on the growth conditions.

One Be related transition with low intensity was observed at $3.35 \mathrm{eV}$, as shown in Fig. 1(a). The intensity of this line varied as a function of the lateral quality of the GaN sample. We attribute this line to $e A$ recombinations. The ionization energy of Be acceptors was calculated to be $150 \pm 10$ $\mathrm{meV}$, assuming a band gap of $3.5 \mathrm{eV}$ for GaN. This value is approximately twice that calculated by Bernardini et al. ${ }^{3}$; it is also about half the ionization energy of $\mathrm{Mg}$ acceptors in $\mathrm{GaN}$. Temperature dependent PL measurements showed different intensity behaviors of the lines at 3.444 and $3.35 \mathrm{eV}$; therefore, we can exclude that the line at $3.35 \mathrm{eV}$ is a phonon replica of the $3.444 \mathrm{eV}$ line. Furthermore, we never observed the line at $3.35 \mathrm{eV}$ after implantation of $\mathrm{Li}, \mathrm{Si}, \mathrm{Ge}$, In, and $\mathrm{Er}$ even when the line at $3.444 \mathrm{eV}$ was present. ${ }^{14}$ These observations prove that the line at $3.35 \mathrm{eV}$ is only related to the implanted Be acceptors.

Other authors ${ }^{15-18}$ have observed a PL line at $3.364 \mathrm{eV}$ which is very close to the $\mathrm{Be}$ acceptor line and which always appeared together with another PL line at $3.305 \mathrm{eV}$. Both lines (commonly labeled as $I_{3} / I_{4}$ or $L_{3} / L_{4}$ ) have been attributed to dislocations, ${ }^{15}$ cubic $\mathrm{GaN},{ }^{16}$ or impurities. ${ }^{17,18} \mathrm{We}$ also initially observed these two lines together with their phonon replicas; however, we determined them to be luminescence lines of the underlying sample holder consisting of a copper alloy. We observed them with varying intensity by exciting the sample holder at low temperatures with the $\mathrm{He}-\mathrm{Cd}$ laser. The respective "phonon replicas" reported in Downloaded 16 Apr 2008 to 152.1.24.180. Redistribution subject to

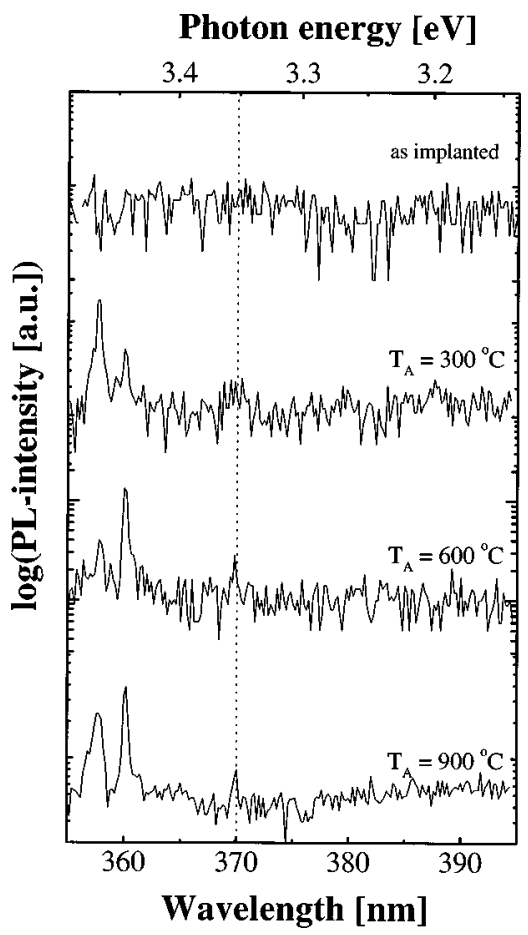

FIG. 2. PL spectra of Be implanted GaN [same dose implanted sample as shown in Fig. 1(a)] as a function of annealing temperature on a logarithmic scale.

Refs. 15-18 originate from elastic photon-phonon scattering processes in the GaN sample reflecting the real GaN phonon energy around $85 \mathrm{meV}$.

Figure 2 shows the recorded PL spectra for the same Be implanted GaN sample presented in Fig. 1(a) as a function of annealing temperature. No lines were observed directly after ion implantation for this dose, but they were observed in the case of lower implantation doses. After annealing to $300{ }^{\circ} \mathrm{C}$ the $I_{2}$ line of the $\mathrm{GaN}$ and the line at $3.444 \mathrm{eV}$ were observed for this sample. The intensities of these PL lines slightly increased as a function of annealing temperature for all implantation doses; however, they were still orders of magnitude lower compared to unimplanted GaN. The implanted material was therefore only partly recovered at this annealing temperature which is in agreement with Ref. 10.

The Be related PL line at $3.35 \mathrm{eV}$ appeared after annealing to $600{ }^{\circ} \mathrm{C}$ and slightly increased after the $900{ }^{\circ} \mathrm{C}$ anneal for the implantation dose of $5 \times 10^{13} \mathrm{~cm}^{-2}$. For a lower implantation dose this line was already observed in the asimplanted samples. For higher implantation doses, higher annealing temperatures were necessary.

The dose dependence is shown in more detail in Fig. 3. PL spectra were obtained after annealing to $900{ }^{\circ} \mathrm{C}$ for the Be implanted GaN samples without (a) and with (b) coimplantation of nitrogen. Weaker luminescence lines were observed in the samples subjected to the higher doses, as there were no radiative paths dominating the recombination of electrons in the highly defective implanted GaN. However, in all implanted GaN samples the Be related PL line at $3.35 \mathrm{eV}$ was present with the exception of the high dose co-implanted sample.

No significant changes in the PL spectra of the $\mathrm{N}+\mathrm{Be}$ co-implanted samples were observed relative to the single Be implanted GaN materials, as shown in Fig. 3. Only a reduc-
AlP license or copyright; see http://apl.aip.org/api/copyright.jsp 
Photon energy [eV]

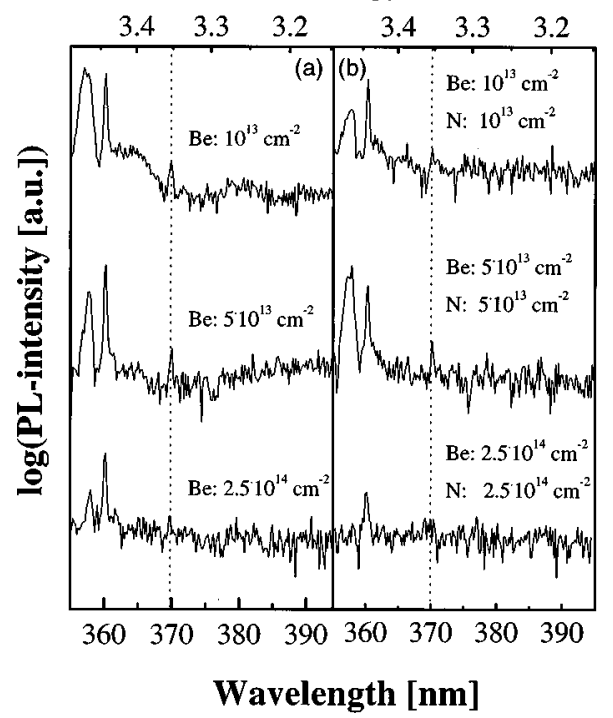

FIG. 3. Implantation dose dependent PL spectra obtained from Be implanted $\mathrm{GaN}$ after annealing to $900^{\circ} \mathrm{C}$ without (a) and with (b) coimplantation of nitrogen.

tion of all lines was observed due to the additional implantation damage.

In conclusion, we present for the first time experimental evidence that Be acceptors are optically active after implantation of this element into GaN. The measured ionization energy of $150 \pm 10 \mathrm{meV}$ confirms that isolated $\mathrm{Be}$ has the most shallow acceptor level in GaN. Co-implantation of nitrogen did not enhance the activation of the Be acceptors.

The authors would like to thank Cree Research, Inc. for supplying the $\mathrm{SiC}$ substrates for this research. The implantations were conducted by ImplantSciences, Wakefield, MA. This work was supported by the ONR under Contract No.
N00014-92-J-1477 (M. Yoder, technical monitor). One of the authors, C. R., is grateful for funding by the DFG (Ro 1198/2-1). R. Davis was supported in part by the Kobe Steel, Ltd. Professorship.

${ }^{1}$ U. Kaufmann, M. Kunzer, M. Maier, H. Obloh, A. Ramakrishnan, B. Santic, and P. Schlotter, Appl. Phys. 72, 1326 (1998).

${ }^{2}$ T. W. Weeks, Jr., M. D. Bremser, K. S. Ailey, E. P. Carlson, W. G. Perry, and R. F. Davis, Appl. Phys. Lett. 67, 401 (1995); J. Mater. Res. 11, 1011 (1996).

${ }^{3}$ F. Bernardini, V. Fiorentini, and A. Bosin, Appl. Phys. Lett. 70, 2990 (1997).

${ }^{4}$ O. Brandt, H. Yang, H. Kostial, and K. H. Ploog, Appl. Phys. Lett. 69, 2707 (1996).

${ }^{5}$ A. Salvador, W. Kim, Ö. Aktas, A. Botchkarev, Z. Fan, and H. Morkoç, Appl. Phys. Lett. 69, 2692 (1996).

${ }^{6}$ T. S. Cheng, C. T. Foxon, L. C. Jenkins, S. E. Hooper, D. E. Lacklison, J. W. Orton, B. Y. Ber, A. V. Merkulov, and S. V. Novikov, Semicond. Sci. Technol. 11, 538 (1996).

${ }^{7}$ R. G. Wilson, S. J. Pearton, C. R. Abernathy, and J. M. Zavada, Appl. Phys. Lett. 66, 2238 (1995).

${ }^{8}$ R. G. Wilson, C. B. Vartuli, S. J. Pearton, C. R. Abernathy, and J. M. Zavada, Solid-State Electron. 38, 1329 (1995).

${ }^{9}$ J. C. Zolper, J. Cryst. Growth 178, 157 (1997), and references therein.

${ }^{10}$ C. Ronning, M. Dalmer, M. Deicher, M. Restle, H. Hofsäss, M. D. Bremser, and R. F. Davis, Mater. Res. Soc. Symp. Proc. 468, 407 (1997), and references therein.

${ }^{11}$ J. I. Pankove and J. A. Hutchby, J. Appl. Phys. 47, 5387 (1976).

${ }^{12}$ J. F. Ziegler, J. P. Biersack, and U. Littmark, The Stopping and Ranges of Ions in Solids (Pergamon, New York, 1985).

${ }^{13}$ S. Fischer, C. Wetzel, E. E. Haller, and B. K. Meyer, Appl. Phys. Lett. 67, 1298 (1995)

${ }^{14}$ E. P. Carlson, C. Ronning, and R. F. Davis (unpublished).

${ }^{15}$ C. Wetzel, S. Fischer, J. Krüger, E. E. Haller, R. J. Molnar, T. D. Moustakas, E. N. Mokhov, and P. G. Baranov, Appl. Phys. Lett. 68, 2556 (1996).

${ }^{16}$ C. H. Hong, D. Pavlidis, S. W. Brown, and S. C. Rand, J. Appl. Phys. 77, 1705 (1995).

${ }^{17}$ W. Götz, N. M. Johnson, J. Walker, D. P. Bour, and R. A. Street, Appl. Phys. Lett. 68, 667 (1996).

${ }^{18}$ D. Haase, M. Burkard, M. Schmidt, A. Dören, H. Schweizer, H. Bolay, and F. Scholz, Mater. Sci. Forum 258-263, 1093 (1997). 